\title{
GRB 050717: A LONG, SHORT-LAG, HIGH-PEAK ENERGY BURST OBSERVED BY SWIFT AND KONUS
}

\author{
H. A. Krimm, ${ }^{1,2}$ C. Hurkett ${ }^{3}$ V. Pal'shin, ${ }^{4}$ J. P. Norris, ${ }^{1}$ B. Zhang, ${ }^{5}$ S. D. Barthelmy, ${ }^{1}$ D. N. Burrows, ${ }^{6}$ \\ N. Gehrels, ${ }^{1}$ S. Golenetskit, ${ }^{4}$ J. P. Osborne, ${ }^{3}$ A. M. Parsons, ${ }^{1}$ M. Perri, ${ }^{7}$ and R. Willingale ${ }^{3}$ \\ Received 2005 December 23; accepted 2006 May 19
}

\begin{abstract}
The long burst GRB 050717 was observed simultaneously by the Burst Alert Telescope (BAT) on Swift and the Konus instrument on Wind. Significant hard to soft spectral evolution was seen. Early gamma-ray and X-ray emission was detected by both BAT and the X-Ray Telescope (XRT) on Swift. The XRT continued to observe the burst for 7.1 days and detect it for 1.4 days. The X-ray light curve showed a classic decay pattern; the afterglow was too faint for a jet break to be detected. No optical, infrared, or ultraviolet counterpart was discovered despite deep searches within $14 \mathrm{hr}$ of the burst. Two particular features of the prompt emission make GRB 050717 a very unusual burst. First, the peak of the $\nu F(\nu)$ spectrum was observed to be $2401_{-568}^{+781} \mathrm{keV}$ for the main peak, which is the highest value of $E_{\text {peak }}$ ever observed. Second, the spectral lag for GRB 050717 was determined to be $2.5 \pm 2.6 \mathrm{~ms}$, consistent with zero and unusually short for a long burst. This lag measurement suggests that this burst has a high intrinsic luminosity and hence is at high redshift $(z>2.7)$. Despite these unusual features, GRB 050717 exhibits the classic prompt and afterglow behavior of a gamma-ray burst.
\end{abstract}

Subject heading: gamma rays: bursts

\section{INTRODUCTION}

It has long been realized that the full understanding of gammaray bursts (GRBs) requires multiwavelength observations as close together in time as possible. The unique capabilities of the Swift Gamma-Ray Burst Explorer (Gehrels et al. 2004) allow such observations to be carried out rapidly and with high sensitivity at $\mathrm{X}$-ray energies ranging from 0.3 to $\sim 350 \mathrm{keV}$. When a GRB is also detected simultaneously with the Konus instrument (Aptekar et al. 1995) on Wind, one also obtains spectral and temporal data up to $>10 \mathrm{MeV}$, providing a complete picture of the prompt emission over nearly 2 orders of magnitude in energy.

When a spectroscopic redshift is not available, it is possible to use features of the prompt emission to constrain estimates of the burst redshift. In particular, Norris et al. (2000) noted that pulse peaks migrate to later times as they become wider at low energies. This spectral lag was found to be proportional to the total peak luminosity of the burst, and can be used along with the peak flux and the peak of the $\nu F(\nu)$ spectrum, or $E_{\text {peak }}$ to constrain the absolute luminosity and hence the redshift of the burst. Also, Amati et al. (2002) and Yonetoku et al. (2004) have shown that $E_{\text {peak }}$ when converted to the GRB rest frame is proportional to isotropic energy (Amati et al. 2002) or peak luminosity (Yonetoku et al. 2004).

After a few hundred seconds, the prompt gamma-ray emission has decayed and the spectrum has softened to the point where high-energy photons are no longer detectable. However, with a sensitive instrument such as the X-Ray Telescope (XRT) on Swift,

\footnotetext{
1 NASA Goddard Space Flight Center, Greenbelt, MD 20771.

2 Universities Space Research Association, 10211 Wincopin Circle, Suite 500, Columbia, MD 21044-3432.

3 Department of Physics and Astronomy, University of Leicester, Leicester LE1 7RH, UK.

4 Ioffe Physico-Technical Institute, Laboratory for Experimental Astrophysics, 26 Polytekhnicheskaya, St. Petersburg 194021, Russia.

5 Physics Department, University of Nevada, Las Vegas, NV 89154.

6 Department of Astronomy and Astrophysics, 525 Davey Laboratory, Pennsylvania State University, University Park, PA 16802.

7 ASI Science Data Center, Via Galileo Galilei, I-00044 Frascati, Italy.
}

this late phase can often be detected in X-rays for many days after the initial burst. Several authors (Zhang et al. 2006; Nousek et al. 2006; Panaitescu et al. 2006) have presented a unified picture of the time evolution of the early X-ray emission. In this unified picture, the initial decay component has a steep time decay function where the emission is dominated by the tail of the internal shock emission (Kumar \& Panaitescu 2000), followed by a shallower component where the fireball has decelerated and emission is dominated by the forward shock (Mészáros \& Rees 1997; Sari et al. 1998).

The long, bright GRB 050717 was detected by both the Burst Alert Telescope (BAT) (Barthelmy et al. 2005a) on Swift (Hurkett et al. 2005a) and the Konus instrument on Wind (Golenetskii et al. 2005), allowing simultaneous observations from $14 \mathrm{KeV}$ to $14 \mathrm{MeV}$. The burst was long enough that it was still detectable in Swift BAT for $>60 \mathrm{~s}$ after it became visible to the Swift XRT. The XRT continued to observe the afterglow until 7.1 days after the trigger, and it was detectable out to 1.4 days. No optical transient was found in spite of deep long-wavelength searches within $14 \mathrm{hr}$ of the GRB.

In this paper we describe the prompt and afterglow properties of GRB 050717, starting with a description of the Swift, Konus, and various optical follow-up observations in $\S 2$, and continuing in $\S 3$ with a discussion of the light curves and spectroscopy from the prompt through the late postburst phase. In $\S 4$ we discuss the implications of these observations, and in particular note the extremely high value of $E_{\text {peak }}$ and unusually short value of the spectral lag.

We show that while GRB 050717 is a classical long GRB, based on its spectral and temporal properties, it exhibits several highly unusual and noteworthy features that may constrain burst models.

\section{OBSERVATIONS AND DATA ANALYSIS}

\subsection{Swift BAT}

At 10:30:52.21 UT, 2005 July 17, the BAT located on board Swift triggered on GRB 050717 (BAT trigger 146372; Hurkett et al. 2005a). Unless otherwise specified, times in this article are 
referenced to the BAT trigger time (UT 10:30:52.21), hereafter designated $T_{0}$. The burst was detected in the part of the BAT field of view that was $55 \%$ coded, meaning that it was $36^{\circ}$ off-axis and only $55 \%$ of the BAT detectors were illuminated by the source. The spacecraft began to slew to the source location $8.66 \mathrm{~s}$ after the trigger and was settled at the source location at $T_{0}+63.46 \mathrm{~s}$.

The BAT data for GRB 050717 between $T_{0}-300 \mathrm{~s}$ and $T_{0}+$ $300 \mathrm{~s}$ were collected in event mode with $100 \mu$ s time resolution and $\sim 6 \mathrm{keV}$ energy resolution. The data were processed using standard Swift BAT analysis tools and the spectra were fit using XSPEC, version 11.3. Each BAT event was mask tagged using batmaskwtevt with the best-fit source position. Mask tagging is a technique in which each event is weighted by a factor representing the fractional exposure to the source through the BAT coded aperture. A weight of +1 corresponds to a fully open detector and a weight of -1 to a fully blocked detector. Flux from the background and other sources averages to zero with this method. All of the BAT GRB light curves shown have been background subtracted by this method. This method is effective even when the spacecraft is moving, since complete aspect information is available during the maneuver.

The mask weighting is also applied to produce weighted, background-subtracted counts spectra using the tool batbinevt. Since the response matrix depends on the position of the source in the BAT field of view, separate matrices are derived for before the slew, after the slew, and for individual segments of the light curve during the slew.

\subsection{Wind Konus}

The long, hard GRB 050717 triggered Wind Konus $(W \mathrm{~K})$ (Aptekar et al. 1995) at $T_{0}(W \mathrm{~K})=10: 30: 57.426 \mathrm{UT}$. It was detected by the S1 detector, which observes the south ecliptic hemisphere; the incident angle was $55^{\circ} .5$. The propagation delay from $S$ wift to Wind is $2.369 \mathrm{~s}$ for this GRB, i.e., correcting for this factor, one sees that the $W \mathrm{~K}$ trigger time corresponds to $T_{0}+$ $2.86 \mathrm{~s}$. The data before $T_{0}(W \mathrm{~K})-0.512 \mathrm{~s}$ were collected in the waiting mode with $2.944 \mathrm{~s}$ time resolution. From $T_{0}(W \mathrm{~K})$ to $T_{0}(W \mathrm{~K})+430.848 \mathrm{~s}, 64$ spectra in 101 channels were accumulated. The first four spectra were accumulated on a $64 \mathrm{~ms}$ timescale, then the spectra accumulation times were varied from 5.120 to $8.192 \mathrm{~s}$ adapting to the current burst intensity. Data were processed using standard Wind Konus analysis tools, and the spectra were fitted by XSPEC, version 11.3. As observed by Wind Konus, GRB 050717 had a steep rise and a long decaying tail.

\subsection{Swift XRT}

The spacecraft slewed immediately to the BAT location of GRB 050717, and the XRT began observing the burst at 10:32:11.49 UT (approximately $79 \mathrm{~s}$ after the BAT trigger). The automated onboard XRT software was unable to centroid on the burst; however, the downlinked X-ray spectrum and light curve clearly showed a bright fading X-ray object in the field. XRT observations (Hurkett et al. 2005b) began in windowed timing (WT) mode (see below) $91 \mathrm{~s}$ after the trigger before going into photon counting (PC) mode at $310 \mathrm{~s}$. The coordinates of the burst were determined by the XRT to be $(\mathrm{J} 2000.0)$ : R.A. $=14^{\mathrm{h}} 17^{\mathrm{m}} 24^{\mathrm{s}} .58$ (214.352), decl. $=$ $-50^{\circ} 31^{\prime} 59^{\prime \prime} .92\left(-50^{\circ} .533\right)$ (the $90 \%$ confidence error circle radius is 3."5; Moretti et al. 2006).

Swift's X-ray Telescope uses a grazing incidence Wolter I telescope to focus X-rays onto a CCD-22 detector. It has an effective area of $135 \mathrm{~cm}^{2}$ at $1.5 \mathrm{keV}$ and an angular resolution of $18^{\prime \prime}$. (For further information on the XRT, see, e.g., Burrows et al. 2004, 2005a; Gehrels et al. 2004; Hill et al. 2004.) This instrument has three key functions: the rapid, automated, and accurate determi-
TABLE 1

UVOT Limiting Magnitudes

\begin{tabular}{|c|c|c|c|}
\hline Filter & $\begin{array}{c}\text { Exposure } \\
\text { (s) }\end{array}$ & $\begin{array}{l}T_{\text {mid }} \\
(\mathrm{s})\end{array}$ & $3 \sigma$ Limit \\
\hline$V$ & 168 & 424 & 19.00 \\
\hline В & 75 & 524 & 19.59 \\
\hline U & 78 & 511 & 19.34 \\
\hline 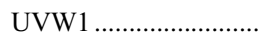 & 78 & 498 & 18.62 \\
\hline 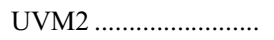 & 78 & 483 & 18.79 \\
\hline UVW2 ….................... & 68 & 498 & 18.73 \\
\hline
\end{tabular}

Notes.-Data taken from GCN 3638 (Blustin et al. 2005). The value $T_{\text {mid }}$ is the midpoint of the summed observation measured with respect to the BAT trigger time $T_{0}$.

nation of GRB positions; the provision of moderate-resolution spectroscopy (energy resolution $140 \mathrm{eV}$ at $5.9 \mathrm{keV}$ ); and recording GRB light curves over a wide dynamic range covering more than 7 orders of magnitude in flux.

The WT readout mode of the XRT uses a restricted portion of the telescope's total field of view: the central 8' (or 200 columns), when the GRB flux is below $\sim 5000$ mcrab. Each column is clocked continuously to provide timing information with $1.8 \mathrm{~ms}$ resolution. However, this rapid readout mode only preserves imaging information in one dimension. Once the GRB flux drops below $\sim 1$ mcrab, the PC mode takes over. This mode retains full imaging and spectroscopic information with a readout time of $2.5 \mathrm{~s}$.

Data for this burst were obtained from the Swift Quick-Look Web site ${ }^{8}$ and processed with version 2 of the Swift software. The XSELECT program was used to extract source and background spectra and cleaned event lists $(0.3-10.0 \mathrm{keV})$, using XSELECT grades $0-12$ for PC mode data and grades $0-2$ for WT data.

The PC mode suffers from pileup when the count rate is $\geq 0.8$ counts $\mathrm{s}^{-1}$. To counter this we extracted a series of grade $0-12$ background-corrected spectra from the first $8.6 \mathrm{ks}$ of PC mode data using annuli of varying inner radii. We deem the point at which pileup no longer affects our results to be when the spectral shape no longer varies with an increase in annular radius. For GRB 050717 this occurred when we excluded the inner 12 pixels (radius). Only the first $500 \mathrm{~s}$ of PC mode data suffered from pileup. The WT data were free from pileup problems. The spectra were then analyzed as normal in XSPEC, version 11.3.21. The light curve was created by the same method as detailed in Nousek et al. (2006).

\subsection{Swift UVOT}

Observations with the Swift Ultra Violet/Optical Telescope (UVOT) began at 10:32:10.7 UT (78 s after the BAT trigger; Blustin et al. 2005). The first datum taken after the spacecraft settled was a $100 \mathrm{~s}$ exposure using the $V$ filter with the midpoint of the observation at $128 \mathrm{~s}$ after the BAT trigger. No new source was detected within the XRT error circle in summed images in any of the six filters down to the $3 \sigma$ magnitude upper limits shown in Table 1.

\subsection{Other Observations}

GRB 050717 was not well positioned for follow-up observations. Its high southern declination made it unobservable by most northern hemisphere telescopes, and the trigger was just before dawn at the South American observatories. Consequently, no

${ }^{8}$ See http://swift.gsfc.nasa.gov/cgi-bin/sdc/ql. 
follow-up optical observations were made until more than $13 \mathrm{hr}$ after the burst. In the several observations that were made after this time, no optical counterpart was detected.

Under the control of Skynet, the Panchromatic Robotic Optical Monitoring and Polarimetry Telescopes (PROMPT) automatically observed the refined XRT localization of GRB 050717 beginning $13.0 \mathrm{hr}$ after the burst (MacLeod et al. 2005). No source was detected within this localization. Limiting magnitudes $(3 \sigma)$, based on five USNO-B1.0 stars, are $21.7\left(R_{c}, T_{0}+\right.$ $13.67 \mathrm{hr})$ and $21.5\left(I_{c}, T_{0}+16.02 \mathrm{hr}\right)$.

Observations in the $K$ band were made with the Wide-Field Infrared Camera on the du Pont 100 inch $(2.5 \mathrm{~m})$ telescope at Las Campanas Observatory on two occasions: 2005 July 18.01 UT $\left(T_{0}+13.7 \mathrm{hr}\right.$; Berger \& Lopez-Morales 2005) and 2005 July 18.98 UT $\left(T_{0}+37.0 \mathrm{hr}\right.$; Berger et al. 2005). Within the $6^{\prime}$ radius XRT error circle four sources were found, of which one is also visible in 2MASS $K$-band images. The other three sources have magnitudes of 18.1,18.7, and 19.2 in comparison to several 2MASS stars; the $3 \sigma$ limiting magnitude of the image is about 19.4. None of the three uncataloged objects faded between the two observations. In addition, Berger et al. (2005) obtained I-band images with the LDSS-3 instrument on the Magellan Clay telescope on 2005 July 18.06 and 18.97 UT (14.9 and $36.8 \mathrm{hr}$ after the burst, respectively). The same three sources visible in the $K$-band images were detected but had not faded.

Luckas et al. (2005) obtained six 5 minute unfiltered images on 2005 July 18.46 UT ( $24.5 \mathrm{hr}$ after the burst), using one of Tenagra Observatory's $0.35 \mathrm{~m}$ telescopes with an AP6 CCD at Perth, Western Australia. No new source was detected within the XRT error circle of GRB 050717 down to the DSS-2R limiting magnitude.

\section{LIGHT CURVES AND SPECTROSCOPY}

\subsection{Swift BAT}

The BAT triggered on the first of two short, small spikes that preceded the main emission of GRB 050717. This first spike at $T_{0}$ was very soft (photon power-law spectral index $2.89 \pm 0.14$ ) and lasted $128 \mathrm{~ms}$. The second short spike began at $T_{0}+0.7 \mathrm{~s}$, was of longer duration $(320 \mathrm{~ms})$, and was much harder (photon index $1.36 \pm 0.23)$. The precursors are shown in detail in the left-hand panels of Figure 1. These small precursors were followed by the main pulse, which displayed the common fast rise, exponential decay (FRED) profile. The intensity rose from background to peak within $450 \mathrm{~ms}$, then began to decay with an average exponential decay constant $1.82_{-0.11}^{+0.13}$. The full light curve is shown in the right-hand panels of Figure 1 . The peak count rate was measured by BAT to be $\sim 16,000$ counts $\mathrm{s}^{-1}$ at $T_{0}+4 \mathrm{~s}$ in the $15-350 \mathrm{keV}$ band. On top of this slow decay, there were at least four other peaks, showing a gradual spectral softening. The duration $T_{90}(15-350 \mathrm{keV})$ is $86 \pm 2 \mathrm{~s}$ (estimated error including systematics). The total fluence in the $15-350 \mathrm{keV}$ band is $(1.40 \pm$ $0.03) \times 10^{-5} \mathrm{ergs} \mathrm{cm}^{-2}$. The $1 \mathrm{~s} \mathrm{peak} \mathrm{photon} \mathrm{flux} \mathrm{measured} \mathrm{from}$ $T_{0}+2.8 \mathrm{~s}$ in the $15-350$ band is $8.5 \pm 0.4$ photons $\mathrm{cm}^{-2} \mathrm{~s}^{-1}$. All the quoted errors are at the $90 \%$ confidence level. The fluence hardness ratio for this burst is $S(100-300 \mathrm{keV}) / S(50-100 \mathrm{keV})=$ $(8.13 \pm 0.14) \times 10^{6} \mathrm{ergs} \mathrm{cm}^{-2} /(2.23 \pm 0.06) \times 10^{6} \mathrm{ergs} \mathrm{cm}^{-2}=$ 3.65 .

The BAT data were binned into 11 time bins to track the spectral evolution of the prompt emission. This is shown in the bottom panels of Figure 1. Starting with the main peak, there is clear evidence of spectral softening as the burst progresses. Then after $T_{0}+91 \mathrm{~s}$, the BAT spectrum hardens again. The fit to the BAT data only over $T_{0}+91$ to $+150 \mathrm{~s}$ yields a power-law photon in-

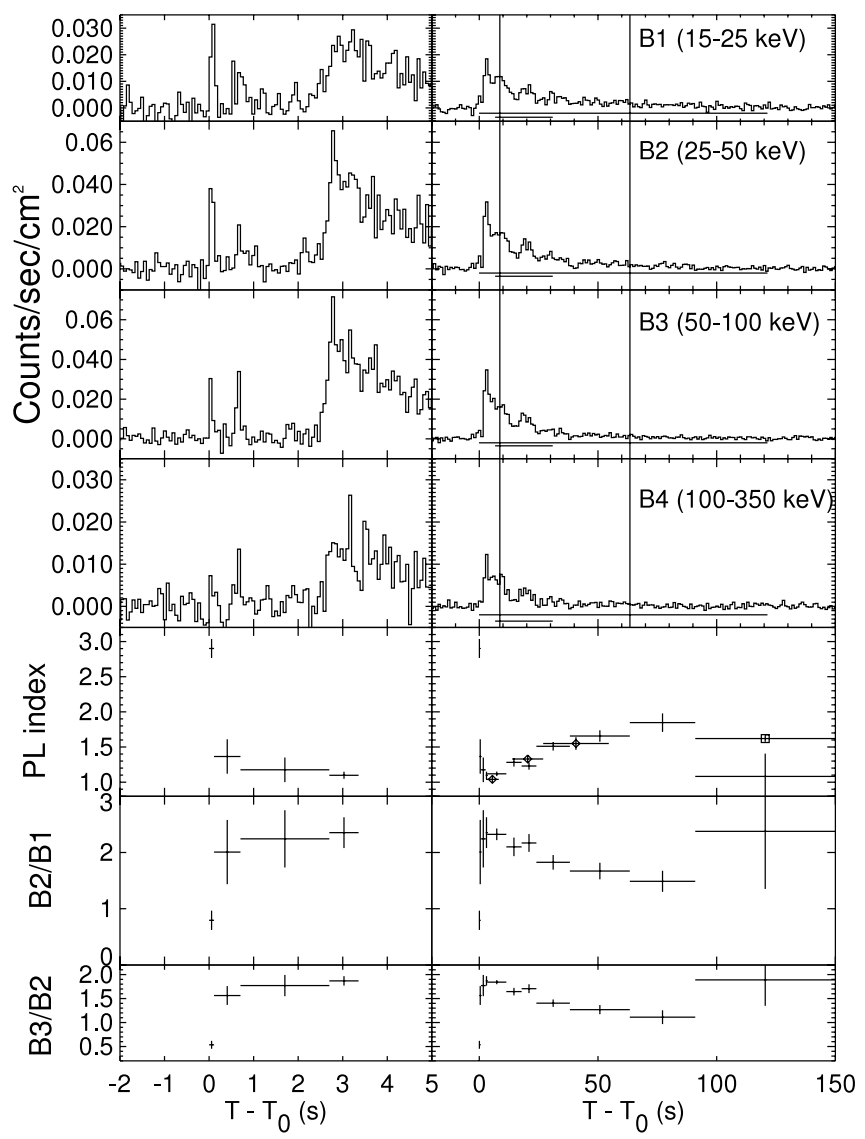

FIG. 1.-Background-subtracted BAT light curves, power-law fit indices, and hardness ratios for GRB 050717. The panels on the right show the full duration of the prompt emission; those on the left zoom in to show the precursor peaks in the light curves more clearly. Light curves (top four sets of plots): Rate is corrected for the effective area as a function of source location in the field of view before and during the slew. After the slew, the source is on-axis. The start and end of the slew to the target are shown by vertical lines. The burst duration measures $T_{90}$ and $T_{50}$ are shown by horizontal lines in the right-hand plots, with $T_{90}$ shown above $T_{50}$. The time binning is $1 \mathrm{~s}$ for the right-hand plots and $64 \mathrm{~ms}$ for those on the left. Power-law fit photon index (bottom plots): Separate fits were made to each time interval indicated. The BAT data (plain symbols) are best fit by a simple power law. The plot also shows joint fits to the BAT and Wind data (diamonds) and to the BAT and XRT data (square). For the leftmost BAT Wind point, the index $\alpha$ of the cutoff power-law fit (see text) is shown. For the other joint fit points, the photon index from a power-law fit is shown. $B A T$ hardness ratios (bottom plots): Two sets of ratios (defined on the plot) are shown to illustrate the spectral hardening during the rise to the main peak, followed by a softening as the prompt emission evolves. The final data points show a second hardening of the spectrum. The timescale is the same for all plots in a vertical column.

dex of $1.08 \pm 0.32$. A joint fit to the BAT and XRT data over the same time period (see $\S 3.3$ ) gives a photon index of $1.61 \pm$ 0.08 . The low BAT flux at these times limits statistically meaningful fits to the entire interval. However, given the spectral variation demonstrated earlier in the burst, it is quite possible that there is spectral evolution occurring at these times as well, and the overall spectral fits should be interpreted with caution.

\subsection{Wind Konus}

The $W \mathrm{~K}$ light curve is shown in three energy bands in Figure 2, and the 21-1300 keV light curve (see Fig. 3) is similar to the Swift BAT light curve. The long decaying tail is clearly seen in G1 band $(21-84 \mathrm{keV})$, marginally seen in $\mathrm{G} 2$ band $(84-360 \mathrm{keV})$, and not seen in $\mathrm{G} 3$ band (360-1370 keV). The G2/G1 ratio demonstrates substantial softening of the tail as compared to the main pulse. 


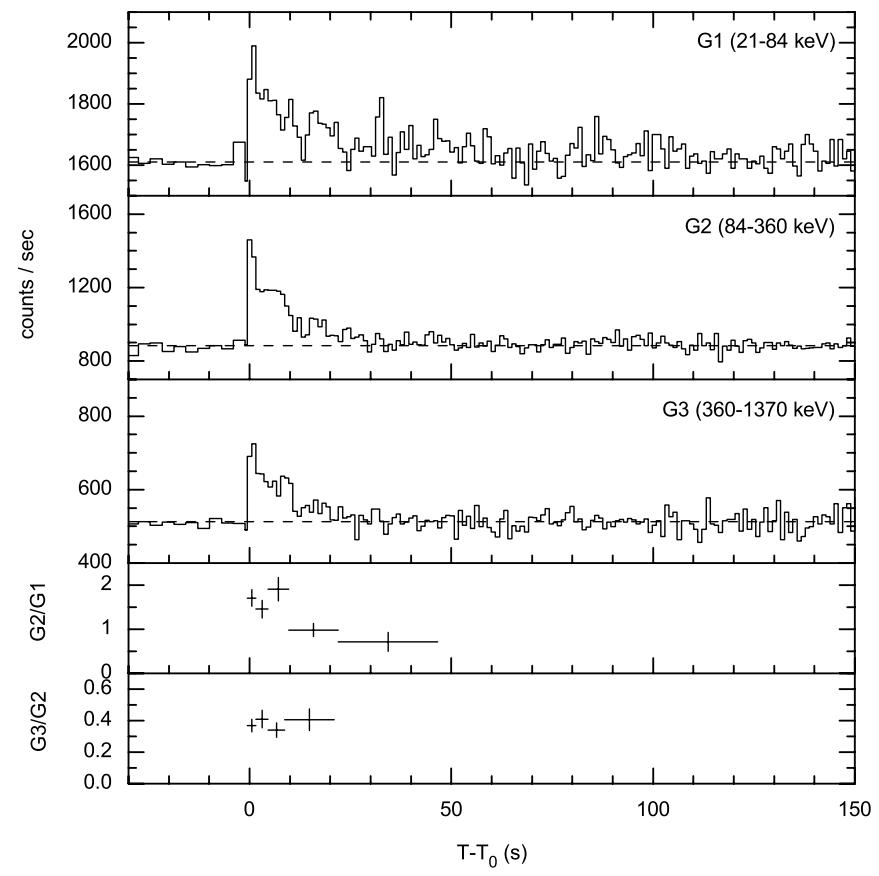

FIG. 2.- The $W \mathrm{~K}$ light curve for GRB 050717 in three energy bands. The data before $T-T_{0}(W \mathrm{~K})=-0.512 \mathrm{~s}$ were recorded in the waiting mode with $2.944 \mathrm{~s}$ time resolution; after that, data were recorded at finer time resolution and binned at $1.024 \mathrm{~s}$. The energy bands used in the hardness ratios at the bottom of the plot are defined in the top panels of the plot.

The $T_{90}$ durations of the burst in G1, G2, and G3 energy bands are $99 \pm 10,95 \pm 11$, and $18 \pm 3 \mathrm{~s}$, respectively. For the sum $\mathrm{G} 1+\mathrm{G} 2+\mathrm{G} 3$, the $T_{90}$ duration is $96 \pm 6 \mathrm{~s}$.

Emission is seen up to $\sim 10 \mathrm{MeV}$. We were able to fit the data in the $20 \mathrm{keV}-6 \mathrm{MeV}$ range by a power-law model with an exponential cutoff: $F(E)=A(E / 100 \mathrm{keV})^{-\alpha} \exp \left[-E(2-\alpha) / E_{\text {peak }}\right]$, where $E$ is the energy in $\mathrm{keV}, E_{\text {peak }}$ is the peak energy of the $\nu F(\nu)$

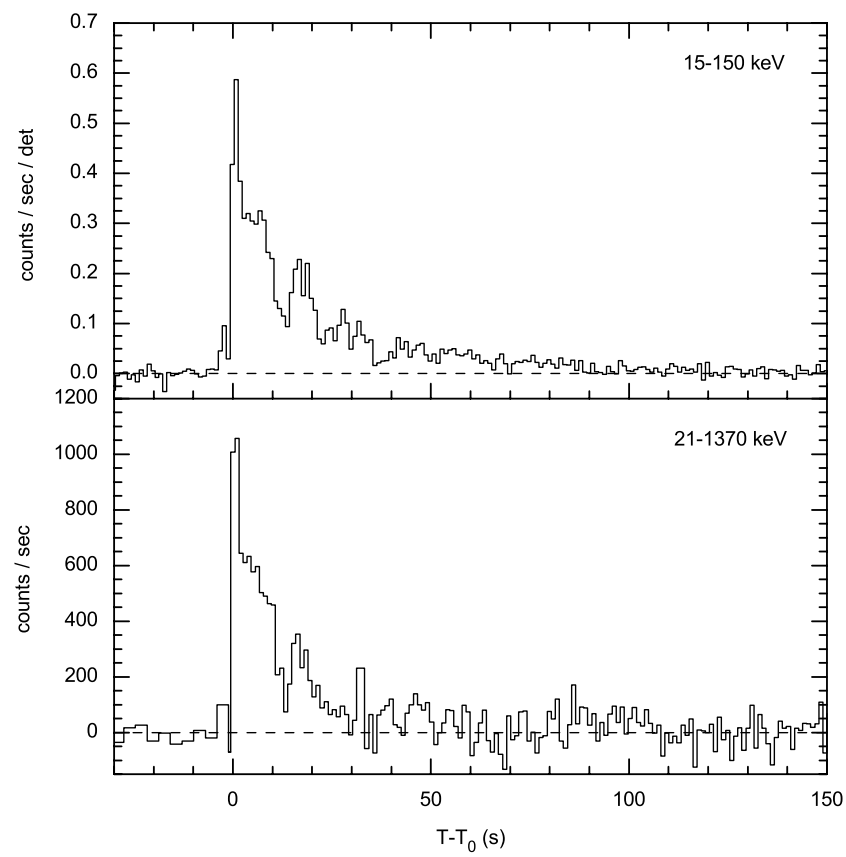

FIG. 3.-Background-subtracted BAT (top) and $W \mathrm{~K}$ (bottom) light curves on the same timescale. The plots have been adjusted so that the trigger time for both plots are the same relative to the burst. This means that $T_{0}$ in the bottom plot is actually $T_{0}(\mathrm{BAT})$ plus the propagation time between the spacecraft $(2.369 \mathrm{~s})$.

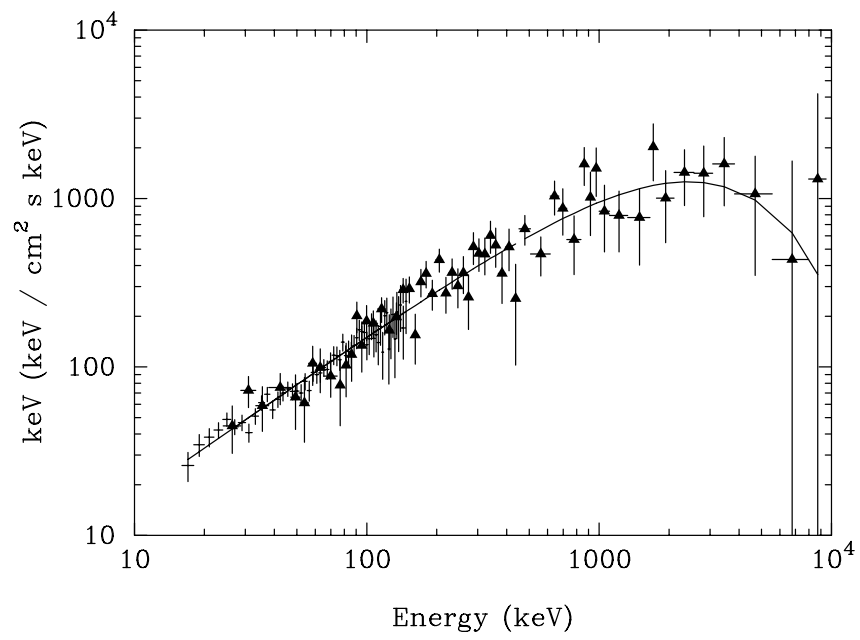

FIG. 4.- Joint fit to a cutoff power-law model (defined in the text) for the BAT and $W \mathrm{~K}$ data during the main peak of emission $T_{0}+2.843$ to $+8.219 \mathrm{~s}$. The value of $E_{\text {peak }}$ for this fit is $2401_{-568}^{+781} \mathrm{keV}$. Points from the BAT spectrum are shown as plus signs; those from the Konus spectrum are shown as triangles.

spectrum, $\alpha$ is the photon index, and $A$ is a normalization factor. For the time-integrated spectrum (from $T_{0}+2.843$ to $54.555 \mathrm{~s}$ ) we find $\alpha=1.19 \pm 0.12$ and $E_{\text {peak }}=2101_{-830}^{+1934} \mathrm{keV}\left(\chi^{2}=88\right.$ for 77 degrees of freedom [dof]). The spectrum of the main peak (from $T_{0}+2.843$ to $+8.219 \mathrm{~s}$ ) is well fitted with $\alpha=1.05 \pm$ 0.10 and $E_{\text {peak }}=2250_{-620}^{+940} \mathrm{keV}\left(\chi^{2}=83\right.$ for 85 dof $)$. Fitting jointly with the BAT data for the main peak gives $\alpha=1.04 \pm$ 0.05 and $E_{\text {peak }}=2401_{-568}^{+781} \mathrm{keV}\left(\chi^{2}=117\right.$ for 143 dof $)$. These values of $E_{\text {peak }}$ for both the time-integrated and time-resolved spectra are perhaps the largest ever measured. The implications of this are discussed in $\S 4.2$. Figure 4 shows that the BAT and Konus data can be well fit to the same model spectrum. A fit to the Band (GRBM) model was also attempted. No statistically significant high-energy power-law tail was established. The limit on the high-energy photon index is $\beta>1.89$ (90\% CL) The lowenergy photon index $\alpha$ is almost the same as for the cutoff powerlaw model, $\alpha=1.02_{-0.3}^{+0.7}$.

Joint fits between BAT and Konus were also made for two later time intervals: $T_{0}+13.851$ to $+26.907 \mathrm{~s}$, and $T_{0}+26.907$ to $+54.555 \mathrm{~s}$. The photon indices for a simple power-law fit are shown in Figure 1. The first of these intervals was also fit with a cutoff power law, but only a lower limit to $E_{\text {peak }}$ was found: $E_{\text {peak }}>548 \mathrm{keV}(90 \% \mathrm{CL})$. We were unable to make a wellconstrained joint BAT-Konus fit to the full burst due to problems creating a single response matrix to cover both the slew and the period before the slew.

The total fluence in the $20 \mathrm{keV}-6 \mathrm{MeV}$ range is $6.5_{-2.2}^{+0.9} \times$ $10^{-5} \mathrm{ergs} \mathrm{cm}^{-2}$. The $64 \mathrm{~ms}$ peak flux measured from $T_{0}+2.86 \mathrm{~s}$ in the same energy band is $1.41_{-0.24}^{+0.18} \times 10^{-5} \mathrm{ergs} \mathrm{cm}^{-2} \mathrm{~s}^{-1}$. The uncertainties in the derived fluence and peak flux are dominated by uncertainties in the high-energy part of the spectrum.

All quoted uncertainties are at the $90 \%$ confidence level.

\subsection{Swift XRT}

The spectrum between 91 and $310 \mathrm{~s}$ after the trigger (WT data) has an average photon index of $1.65 \pm 0.11$, with the absorption fixed at its Galactic value of $2.22 \times 10^{21} \mathrm{~cm}^{-2}$ and an indication of an excess absorption of $(2.75 \pm 0.57) \times 10^{21} \mathrm{~cm}^{-2}$, assuming $z=0$ and standard (local) interstellar material abundances. The mean unabsorbed flux in WT mode at $201 \mathrm{~s}$ (mean time) is $(5.76 \pm 0.31) \times 10^{-10} \mathrm{ergs} \mathrm{cm}^{-2} \mathrm{~s}^{-1}$ in the $0.3-10.0 \mathrm{keV}$ energy range. 


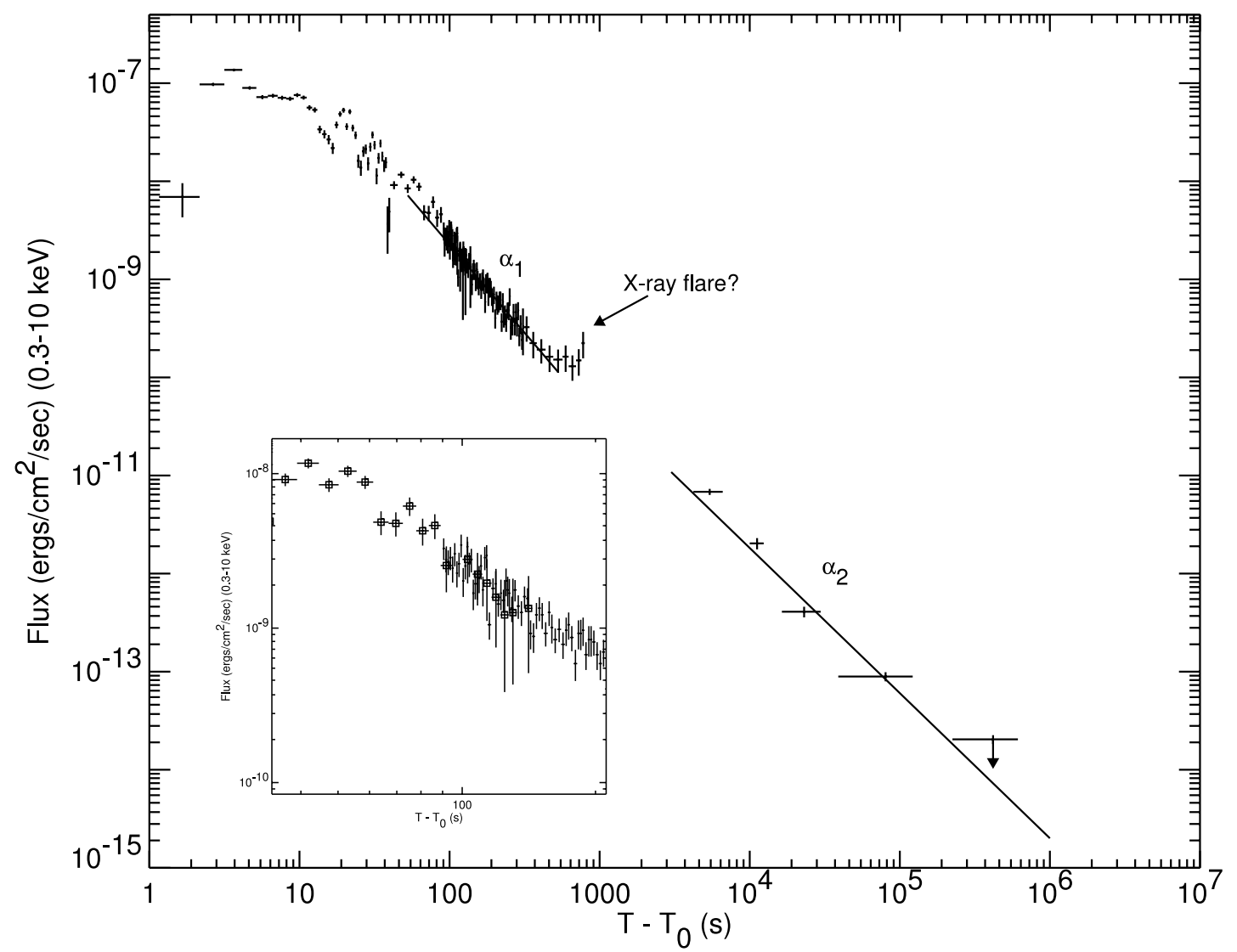

FIG. 5.-Combined BAT prompt emission and XRT afterglow light curve. Points in the BAT light curve have been extrapolated from the BAT $15-150$ keV energy band to the XRT $0.3-10 \mathrm{keV}$ band and corrected for differences in the effective area (see discussion in the text). This shows how the prompt emission makes a smooth transition into the afterglow. The broken power-law fit to the X-ray light curve decay is also shown $\left(\alpha_{1}=2.10 ; \alpha_{2}=1.48\right)$. The last data point (upper limit) was combined from five orbits in PC mode. Inset: Section of Fig. 5 showing the overlap between BAT (squares) and XRT (plus signs) emission. This figure clearly shows the smooth transition from prompt gamma-ray to early X-ray emission. See the text for a discussion of the extrapolation of the BAT data points.

During the period between $T_{0}+91$ and $+150 \mathrm{~s}$, a joint fit was made to the XRT and BAT data. The joint fit gives a photon index of $1.61 \pm 0.08$, with an excess absorption of $3.36_{-0.68}^{+0.8} \times 10^{21} \mathrm{~cm}^{-2}$ $\left(\chi^{2}=125\right.$ for 115 dof $)$. This fit was used to extrapolate the BAT $15-150 \mathrm{keV}$ flux into the XRT energy range $(0.3-10 \mathrm{keV})$ during the overlap interval assuming that the 1.61 power-law index holds in both energy ranges. Since we know the BAT count rate in the BAT $(15-150 \mathrm{keV})$ range, we were able to use XSPEC to derive the model flux in the $0.3-10 \mathrm{keV}$ band and then calculate a ratio between BAT counts $(15-150 \mathrm{keV})$ and flux $(0.3-10 \mathrm{keV})$. For earlier epochs we derived the conversion ratio from the model fits to the BAT data alone. We derived a similar ratio between XRT counts $(0.3-10 \mathrm{keV})$ and flux. With this extrapolation one can directly compare the early and later light curves and show (Fig. 5) that the prompt emission smoothly transitions to the afterglow emission.

The data from $T_{0}+1.17$ to $+8.25 \mathrm{hr}$ were also fit with a power law with a photon index of $1.35 \pm 0.21$ and Galactic absorption $\left(\chi^{2}=16.9\right.$ for $\left.11 \mathrm{dof}\right)$. The model flux over $0.3-10.0 \mathrm{keV}$ was $(1.8 \pm 0.41) \times 10^{-12} \mathrm{ergs} \mathrm{cm}^{-2} \mathrm{~s}^{-1}\left(3.54_{-1.00}^{+0.89} \times 10^{-4}\right.$ photons $\mathrm{cm}^{-2} \mathrm{~s}^{-1}$ ). In this case there was no improvement to the fit by adding excess absorption. Indeed, this later spectrum is not consistent with excess absorption at the level implied by the earlier WT data; the excess absorption is limited at $90 \%$ confidence to $<1.5 \times 10^{21} \mathrm{~cm}^{-2}$.

\subsection{Postburst Emission}

The gamma-ray and X-ray decay light curve is shown in Figure 5. The light curve shows several prominent features that can be interpreted in light of the models discussed in Zhang et al. (2006, hereafter Z06). First, as pointed out earlier, there is a smooth transition from the prompt BAT emission into the early X-ray emission and a fairly steep decay (power-law index $\alpha_{1}$ in the discussion below) until $T_{0}>\sim 200 \mathrm{~s}$. This is followed by a possible superimposed X-ray flare, a phenomenon quite common in GRBs as observed by Swift (Burrows et al. 2005b; Barthelmy et al. 2005b). Unfortunately, observing constraints cut off observations in the middle of the possible flare, and the statistics do not allow for a meaningful fit to a flare component. Observations resumed again at $T_{0}+4214 \mathrm{~s}$, with a return to a power-law decay, with a shallower power-law index ( $\alpha_{2}$ below).

In order to fit the data to reasonable X-ray emission models, two intervals were removed: BAT data points before $T_{0}+50 \mathrm{~s}$, which were believed to be part of the prompt emission, and XRT data points between $T_{0}+500 \mathrm{~s}$ and the end of the first observation, so that the fit is not contaminated by the possible flare. Two different fits were made and are discussed in turn.

First, we tried a broken power law. This gave a power-law index $\alpha_{1}=2.10_{-0.05}^{+0.17}$ for the steep part of the light curve, a break time of $203 \pm 26 \mathrm{~s}$, and an index $\alpha_{2}=1.48 \pm 0.02$ for the shallow part $\left(\chi^{2}=159\right.$ for 111 dof $)$. The steep part of the curve $\left(\alpha_{1}=2.10\right)$ corresponds to region I in Figure 1 of Z06. According to Z06, if this time can be interpreted as the curvature effect (Kumar \& Panaitescu 2000; Dermer 2004), the index should be $\alpha=2+\beta$, where $\beta$ is the energy index of the spectrum of the emission. Taking $\beta=0.62$, we should have $\alpha_{1}=2.62$, as compared to the observed value of 2.10 . 
One factor that could lead to a deviation from the $\alpha=2+\beta$ relation is that the decay curve seen could be a superposition of two separate decay power laws: one steep due to the curvature component, and one shallow due to the forward shock component. So a fit was made to a superposition model: $F(t)=A t^{-\alpha_{1}}+$ $C t^{-\alpha_{2}}$, where $A$ and $C$ are normalization factors. This fit gave a steep index $\alpha_{1}=3.01_{-0.23}^{+0.55}$ and a shallow index $\alpha_{2}=1.43 \pm$ $0.04\left(\chi^{2}=161\right.$ for 110 dof $)$. Statistically this fit is indistinguishable from the broken power law. However, the physical interpretation is more straightforward. The steep index $\left(\alpha_{1}=3.01\right)$ is the decay of the tail of the internal shock emission, which is superimposed on an underlying afterglow component with a decay index of $\alpha_{2}=1.43$. The afterglow component becomes dominant at $T_{0}+\sim 100 \mathrm{~s}$.

It is instructive to compare the measured temporal index $\left(\alpha_{2} \approx\right.$ 1.4) with the values predicted by the simple afterglow models compiled by Zhang \& Mészáros (2004). At late times $(t>$ $1.17 \mathrm{hr}$ ), we should be in the slow cooling regime, and the spectral index of GRB $050717, \beta=0.35 \pm 0.21$, is consistent only with the regime in which $\nu_{m}<\nu<\nu_{c}$. Here, following Zhang \& Mészáros (2004), $\nu$ is the spectral frequency of the emission, and $\nu_{m}$ and $\nu_{c}$ are the synchrotron frequency and cooling frequency, respectively. Using $\beta=0.35 \pm 0.21$, we have the electron-acceleration power-law index $p=1+2 \beta=1.7 \pm 0.4$. Using the equations in Table 1 of Zhang \& Mészáros $(2004)^{9}$ and taking $p>2$, we derive values for $\alpha$ of $0.5 \pm 0.3$, and $1.0 \pm 0.3$ for the ambient interstellar medium (ISM) (Mészáros \& Rees 1997; Sari et al. 1998) and wind models (Chevalier \& Li 2000), respectively. If $1<p<2$ (Dai \& Cheng 2001), we derive $\alpha$ values of $0.7 \pm 0.1,1.2 \pm 0.05$, again for the ISM and wind models, respectively. We see that the late-time temporal index $\left(\alpha_{2} \approx 1.4\right)$ is inconsistent with the ISM model and marginally consistent with the wind model. This analysis shows that at late times emission is dominated by the forward shock with a wind density profile.

In order for the afterglow of GRB 050717 to have $\nu_{m}<\nu<$ $\nu_{c}$, it must be observed at a time such that $t>t_{c}$, where the critical time $t_{c}$ is defined in Z06. This puts constraints on the wind parameter $A_{*}$, which is defined in Chevalier \& Li (2000) as being proportional to the wind mass loss rate divided by the wind velocity (units of grams per centimeter). The parameter $A_{*}$ must be in the range $0.01-0.001$, which is similar to the limit derived for GRB 050128 (Campana et al. 2005).

The late-time shallow decay (index $\sim 1.4$ ) continues until the flux becomes unobservable to the XRT. A lower limit is set for summed observations after $T_{0}+2.6$ days. Since there is no apparent break to a steeper decay in the light curve, the lower limit on a jet break time is $t_{b}>1.4$ days.

\section{DISCUSSION}

\subsection{Spectral Lag}

It is possible to derive an estimate of the spectral lag of the BAT data between channel $2(25-50 \mathrm{keV})$ and channel 4 (100$350 \mathrm{keV})$. From the spectral lag we can use the methodology of Norris et al. (2000) and Norris (2002) to derive limits on the redshift of the GRB and on the isotropic luminosity of the peak of the emission. The spectral lag was derived for the main peak of emission (from $T_{0}+2.26$ to $+5.8 \mathrm{~s}$ ). The lag was found to be $2.5_{-2.4}^{+2.9} \mathrm{~ms}$. Hence, the measured lag is statistically consistent with zero. The lag was also measured for several other intervals during

\footnotetext{
9 We have changed the signs of $\alpha$ and $\beta$ in the equations of Zhang \& Mészáros (2004) to conform to the definition $F_{\nu} \propto t^{-\alpha} t^{-\beta}$ used in this paper.
}

the burst and with time rebinning ranging from 2 to $16 \mathrm{~ms}$. In all cases, the measured lag was small, positive, and consistent with zero. Such a low value for the lag is quite unique for a long burst, since Norris (2002) has shown that the dynamic range of lags for long bursts spans $\sim 25$ to $\sim 300 \mathrm{~ms}$. In fact, out of the 90 brightest bursts studied by Norris (2002), only $2 \%$ show a lag as small as that of GRB 050717.

One can use the lag, the measured peak flux, and $E_{\text {peak }}$ to set lower limits on the distance to the burst. Using the peak flux of $(1.69 \pm 0.16) \times 10^{-6}$ ergs $\mathrm{cm}^{-2} \mathrm{~s}^{-1}\left(15-350 \mathrm{keV} ; T_{0}+2.752\right.$ to $+3.008 \mathrm{~s}$ ), the parameters from the joint Konus-BAT fits to the main peak ( $\S 3.2)$ and the $+2 \sigma$ limit on the lag $(8.3 \mathrm{~ms})$, one derives a redshift of 2.7 and a peak luminosity of $3.9 \times 10^{53} \mathrm{ergs} \mathrm{s}^{-1}$ $(15-350 \mathrm{keV})$. The fit is relatively insensitive to variations in either peak flux or $E_{\text {peak }}$ and other spectral fit parameters. Since smaller values of spectral lag would lead to larger redshifts, this value, $z=2.7$, can be considered the $2 \sigma$ lower limit on the redshift; similarly, the luminosity is also a lower limit. Such a large redshift is consistent with the nondetection of an optical or infrared counterpart to the afterglow ( $\S \S 2.5$ and 4.3) and with the nondetection of a jet break (§3.4).

A consistent interpretation of such a small lag is that the highenergy emission from GRB 050717 has been redshifted downward more than usual into the BAT energy range. It has been shown (Norris et al. 1996; Fenimore \& Bloom 1995) that the high-energy component of burst emission shows narrower peaks and more variation than is seen at lower energies. Shifting such spiky peaks into the BAT range would cause the measured lag to be smaller than what would be observed in long bursts at lower redshifts.

Norris \& Bonnell (2006) have pointed out that many short bursts seen by BATSE, Swift, WK, and HETE-2 have extended emission starting a few seconds after the short spike and lasting for $\sim$ tens of seconds. Since short bursts are also known to have short lags (Norris et al. 2001), is it possible that GRB 050717 is in fact a short burst or a magnetar flare from a nearby galaxy? This burst has a pair of precursors of duration 128 and $320 \mathrm{~ms}$, followed by $>100 \mathrm{~s}$ of extended emission along with a spectral lag consistent with short GRBs. However, two properties of GRB 050717 argue strongly against it being a short burst. First of all, the spectra of the precursors of this burst are significantly softer than the extended emission (see Fig. 1), while in all short bursts with extended emission the short spikes are significantly harder than the extended emission. Second, in GRB 050717, the flux is dominated by the extended emission, while in short bursts, the flux is dominated by the short episode of emission. The ratio of peak to tail emission for a magnetar (Palmer et al. 2005) is even more extreme. Therefore, it is more likely that GRB 050717 is indeed a long burst seen at a large distance.

Using a relationship derived by Liang \& Zhang (2005) we can use the measured $E_{\text {peak }}$ and the limits on luminosity and redshift to set a lower limit on the jet break time for this burst. After rearranging equation (5) in Liang \& Zhang (2005),

$$
t_{b}=0.88\left(E_{\gamma, \text { iso, } 52}\right)^{-0.81}\left(\frac{E_{p}}{100 \mathrm{keV}}\right)^{1.56}(1+z)^{2.56} \text {. }
$$

Here $t_{b}$ is the jet break time in days in the observer frame, $E_{\gamma}$, iso, $52>100$ is the isotropic energy in units of $10^{52} \mathrm{ergs}$, and $E_{p}=2400 \mathrm{keV}$ is the observed peak energy. Errors on the exponents in the equation have been suppressed, since the calculation is dominated by errors in the input parameters. With these values and $z=2.7$, we can derive a lower limit on $t_{b}$ of 88 days. As we saw in $\S 3.4$, this is fully consistent with the observations. 


\subsection{Comparison to Other GRBs}

It was noted in $\S 3.2$ that $E_{\text {peak }}$ for GRB 050717 is unusually high. The observed values are $2400 \mathrm{keV}$ for the main peak and $2100 \mathrm{keV}$ for the time-integrated spectrum; when propagated to a rest frame at $z=2.7$, the intrinsic values of $E_{\text {peak }}$ become 8900 and $7800 \mathrm{keV}$, respectively. These values can be compared to previously measured values of $E_{\text {peak }}$ from the Burst and Transient Source Experiment (BATSE) and BeppoSAX. Kaneko et al. (2006a; see also Kaneko et al. 2006b) have performed a systematic spectral analysis of 350 bright GRBs observed by BATSE. Of these bursts, none show an integrated $E_{\text {peak }}$ as large as what was measured for GRB 050717. The highest value calculated was $2039 \mathrm{keV}$ for GRB 971220. Preece et al. (2000) provided time-resolved spectroscopy for 156 bright BATSE bursts. In studying the catalog provided with the Preece et al. (2000) paper, we found only two bursts that had $E_{\text {peak }}>2000 \mathrm{keV}$ in multiple time-resolved spectra. The moderately bright burst GRB 940526B had $E_{\text {peak }}>2000 \mathrm{keV}$ in seven of the nine time-resolved spectra, although Kaneko et al. (2006a) reported that the best-fit $E_{\text {peak }}$ for this burst is $1689 \mathrm{keV}$. One other BATSE burst, GRB 960529, had well-constrained values of $E_{\text {peak }}>2000 \mathrm{keV}$ in a number of its time-resolved spectra and an unconstrained time-integrated value of $E_{\text {peak }}>2000 \mathrm{keV}$. It is clear from Figure 21 in Kaneko et al. (2006a) that only a very small fraction of the 8459 time-resolved spectra fit by Kaneko et al. (2006a) have $E_{\text {peak }}$ values as large as what is found for GRB 050717. It should be noted, however, that the Konus energy range extends farther than does BATSE $(\sim 2 \mathrm{MeV})$, meaning that some bursts with extremely high values of $E_{\text {peak }}$ may not be well constrained in the Kaneko et al. (2006a) or Preece et al. (2000) fits. In addition, none of the 12 BeppoSAX bursts studied in Amati et al. (2002) have $E_{\text {peak }}$ as high as what we report for GRB 050717. Clearly, GRB 050717 is an exceptional case.

It is instructive to ask if GRB 050717 is unusual in other ways. We can compare, for example, the position of GRB 050717 on a hardness-duration plot to other samples. Using $T_{90}=86 \mathrm{~s}$ and the fluence ratio $S(100-300 \mathrm{keV}) / S(50-100 \mathrm{keV})=3.67$, we can see that this burst does not have a particularly high hardness ratio and falls well within the scatter of long bursts in both the BATSE and BAT samples (see, for example, Sakamoto et al. 2006).

One can also use the redshift inferred from the spectral lag (§ 4.1) to see how GRB 050717 fits the relationships that previous authors have derived between $E_{\text {peak }}$ and isotropic energy (Amati et al. 2002) and peak luminosity (Yonetoku et al. 2004). The lower limits on the isotropic radiated energy, the isotropic peak luminosity, and peak energy in the source rest frame corresponding to the limit $z>2.7$ are $E_{\gamma}^{\text {iso }}>1.1 \times 10^{54}$ ergs $(1-$ $10,000 \mathrm{keV}), L_{\max }^{\text {iso }}>9.6 \times 10^{53} \mathrm{ergs} \mathrm{s}^{-1}(30-10,000 \mathrm{keV})$, and $E_{\text {peak }}^{\text {rest }}>7800 \mathrm{keV}$ (for a standard cosmology: $\Omega_{M}=0.3, \Omega_{\Lambda}=$ $\left.0.7, H_{0}=70 \mathrm{~km} \mathrm{~s}^{-1} \mathrm{Mpc}^{-1}\right)$. These values show that GRB 050717 is an outlier on both the Amati et al. (2002) and Yonetoku et al. (2004) relations in the direction of $E_{\gamma}^{\text {iso }}$ and $L_{\max }^{\text {iso }}$ being smaller than the relations would predict given $E_{\text {peak }}^{\text {rest }}=7800 \mathrm{keV}$. It is not possible to adjust the redshift (within reasonable limits: $z<20$ ) to bring GRB 050717 in line with either relation. Thus, we must conclude that GRB 050717 does not fit either the Amati et al. (2002) or Yonetoku et al. (2004) relations. We note that the maximum value of $E_{\text {peak }}^{\text {rest }}$ used in the derivation of either relation is $\sim 2000 \mathrm{keV}$, so these relations have not been verified for values of $E_{\text {peak }}$ as large as that of GRB 050717.

There have also been a number of recent papers (Band \& Preece 2005; Nakar \& Piran 2005a, 2005b; Kaneko et al. 2006a) that have presented strong evidence that the Amati relation is not universal and that there are many BATSE GRBs for which the relation is inconsistent. GRB 050717 is an excellent example demonstrating that the Amati et al. (2002) relation does not hold for all bursts, particularly those with high $E_{\text {peak }}$. Maybe GRB 050717 and similar bursts will eventually be able to tell us why the Amati relationship is breaking down.

\subsection{Lack of Optical Counterpart}

As noted in $\S \S 2.4$ and 2.5, no optical counterpart to GRB 050717 was found. The deepest limits were those obtained from PROMPT, at $21.7\left(R_{c}, T_{0}+13.67 \mathrm{hr}\right)$ and $21.5\left(I_{c}, T_{0}+16.02 \mathrm{hr}\right)$. What conclusions can be drawn from the lack of an infrared counterpart?

First of all, is GRB 050717 a dark burst? Jakobsson et al. (2004) made a comparison between the observed X-ray flux and the $R$-band magnitude of the afterglow at $10 \mathrm{hr}$ after the trigger for a large set of bursts and defined a dark burst as a burst lying in a certain region of the $\log \left(F_{\text {opt }}\right)-\log \left(F_{\mathrm{X}}\right)$ diagram. For GRB 050717 , the X-ray flux interpolated to $T_{0}+10 \mathrm{hr}$ is $0.015 \mu \mathrm{Jy}$ (see Fig. 5), and the $R$-band limit extrapolated to $T_{0}+10 \mathrm{hr}$ would be $R_{c} \approx$ 21.5. This is solidly within the bright burst region of the Jakobsson et al. (2004) diagram; thus, it is not possible to say that this is a dark burst given how late the optical limits are.

Similarly, the lack of a counterpart cannot be used as confirmation of the high redshift. Assuming $z=2.7$, the Lyman edge would be redshifted to $91.2 \mathrm{~nm}(z+1)=337 \mathrm{~nm}$. This is consistent with the relatively shallow ultraviolet limits set by UVOT, but the counterpart could still easily be observed in the $I$ band. It is instructive to compare the infrared observations of GRB 050717 to those of GRB 050904, a high-redshift $(z=6.29)$ burst for which an infrared counterpart was found. However, the IR observations of GRB 050904 were either much earlier $(J \sim 17.5$, $T_{0}+\sim 3 \mathrm{hr}$; Haislip et al. 2005) or much deeper $(I \sim 22.9 \pm$ $0.6, T_{0}+\sim 37 \mathrm{hr}$; Perley et al. 2005) than those obtained for GRB 050717. The lack of an observed counterpart to GRB 050717 must be attributed to the lateness of the observations.

\section{CONCLUSIONS}

The long gamma-ray burst GRB 050717 shows a number of interesting features that can be interpreted in light of the predominant models of bursts and their afterglows.

It has been known for many years that spectral evolution operates in long GRBs in several ways. Golenetskii et al. (1983) first recognized that the more intense portions of bursts are spectrally harder than the less intense time periods. Concomitantly, individual burst pulses are asymmetric, especially at low energies. This was pointed out by Norris et al. (1996), and later Band (1997) and Norris (2002) showed via spectral lag analysis that, if the burst was bright enough, positive lags were manifest, averaged over the whole time profile. Similarly, and related to the first two effects, the burst "envelope" (containing the peaks and valleys in a burst) tends to soften with time in the vast majority of bursts, an effect that was quantified by Band \& Ford (1998). Nemiroff et al. (1994) tied these effects together by demonstrating conclusively that on all timescales, GRBs are time asymmetric. Thus, the later, usually lower intensity portions of a burst should also be spectrally softer.

The long gamma-ray burst GRB 050717 shows all aspects of these evolutionary trends including overall hard to soft spectral evolution as the prompt emission decays and time asymmetries in all peaks at all energies. Features include two short, soft precursor spikes and at least seven peaks in the main burst. 
The main emission of the burst clearly exhibits hard to soft spectral evolution, as discussed in Zhang \& Mészáros (2004) and Norris et al. (1986). The light curve of the prompt emission (Fig. 1) begins with two short, faint, spectrally soft spikes, followed by an intense peak that is the hardest portion of the burst. The burst intensity envelope as seen above $15 \mathrm{keV}$ decays over the next $\sim 150$ s until it becomes detectable only at lower energies. Superimposed on the overall decay are at least four subsidiary peaks, each of which is less intense and softer than the one before. However, the spectra of the peaks are harder than the intervening valleys. Furthermore, as seen in Figures 1 and 3, each peak is time asymmetric at all energies. Thus, the time profile of this burst is a very good example of the overall time asymmetry described by Nemiroff et al. (1994).

Norris et al. (1996) also showed that the structure of pulses in GRBs is narrower at high energies. This is another aspect of what Norris et al. (1996) has called the "pulse paradigm" and is physically related to the overall spectral evolution of pulses. GRB 050717 was unusual in that its spectral lag is very short (positive but statistically consistent with zero - see $\S 4.1$ ), while nearly all long bursts clearly show a large positive spectral lag (Norris 2002). The short lag and observed brightness of the burst suggest that it is at a high redshift $(z>2.7)$ and hence has a large intrinsic luminosity $\left(L_{\text {peak }}>9 \times 10^{53} \mathrm{ergs} \mathrm{s}^{-1}\right)$. The features observed in the burst are likely representative of spiky high-energy features redshifted to the BAT energy range.

The late decay of GRB 050717 is consistent with a steep decay from the tail of the internal shock emission superimposed on a less steep underlying afterglow component. At later times after the fireball has decayed, the emission is dominated by the forward shock component with an inferred X-ray flare, followed by a shallow decay.
GRB 050717 also demonstrates many of the features of the unified picture of the late-time evolution of GRB emission (Z06; Nousek et al. 2006; Panaitescu et al. 2006). When the BAT flux is extrapolated to the $0.3-10 \mathrm{keV}$ energy range, it is seen that the prompt emission smoothly transitions into the slowly decaying phase. During the early X-ray emission of GRB 050717, the decay index is somewhat less steep than would be expected if it were due solely to the tail emission of the prompt GRB. As discussed in $\S 3.4$, this can be interpreted as a superposition of tail and external shock emission, although other interpretations are also discussed. Before data collection was cut off by an orbital constraint at $\sim 800 \mathrm{~s}$ after the trigger, the light curve shows evidence of the start of an X-ray flare. When observations take up again, the flux is much weaker and the decay index is shallow, since at this time the afterglow is dominated by the forward shock. The flux became too faint to observe before the expected jet break at $t_{b}>90$ days.

The short spectral lag and high $E_{\text {peak }}$ are very unusual for long GRBs, putting GRB 050717 within the bottom $2 \%$ of long bursts for spectral lag and within the highest few bursts detected in terms of peak energy. Other burst and afterglow properties are common and easily interpreted. This is an indication that these properties also hold for bright, high-redshift bursts.

H. A. K. was supported in this work by the Swift project, funded by NASA. This work is supported at the University of Leicester by the Particle Physics and Astronomy Research Council (PPARC). C. P. H. gratefully acknowledges support from a PPARC studentship. The Wind Konus experiment is supported by Russian Space Agency contract and RFBR grant 06-02-16070. H. A. K. is also grateful for useful discussions with T. Sakamoto, P. O’Brien, and Y. Kaneko.
Amati, L., et al. 2002, A\&A, 390, 81

Aptekar, R. L., et al. 1995, Space Sci. Rev., 71, 265

Band, D. L. 1997, ApJ, 486, 928

Band, D. L., \& Ford, L. 1998, Adv. Space Res., 22, 1093

Band, D. L., \& Preece, R. D. 2005, ApJ, 627, 319

Barthelmy, S., et al. 2005a, Space Sci. Rev., 120, 143 2005b, Nature, 438, 994

Berger, E., \& Lopez-Morales, M. 2005, GCN Circ. 3639, http://gcn.gsfc.nasa .gov/gcn/gen3/3639.gen3

Berger, E., et al. 2005, GCN Circ. 3643, http://gcn.gsfc.nasa.gov/gcn/gcn3/ 3643.gcn 3

Blustin, A., et al. 2005, GCN Circ. 3638, http:/gcn.gsfc.nasa.gov/gcn/gen3/ 3638.gcn 3

Burrows, D. N., et al. 2004, Proc. SPIE, 5165, 201

2005a, Space Sci. Rev., 120, 165

2005b, Science, 309, 1833

Campana, S., et al. 2005, ApJ, 625, L23

Chevalier, R. A., \& Li, Z.-Y. 2000, ApJ, 536, 195

Dai, Z. G., \& Cheng, K. S. 2001, ApJ, 558, L109

Dermer, C. D. 2004, ApJ, 614, 284

Fenimore, E. E., \& Bloom, J. S. 1995, ApJ, 453, 25

Gehrels, N., et al. 2004, ApJ, 611, 1005

Golenetskii, S., et al. 1983, Nature, 306, 451

2005, GCN Circ. 3640, http://gcn.gsfc.nasa.gov/gcn/gcn3/3640.gcn3

Haislip, J., et al. 2005, GCN Circ. 3913, http://gen.gsfc.nasa.gov/gcn/gcn3/ 3913.gcn 3

Hill, J., et al. 2004, Proc. SPIE, 5165, 217

Hurkett, C., et al. 2005a, GCN Circ. 3633, http:/gcn.gsfc.nasa.gov/gcn/gcn3/ 3633.gcn 3

- 2005b, GCN Circ. 3636, http://gcn.gsfc.nasa.gov/gcn/gcn3/3636.gcn3

Jakobsson, P., et al. 2004, ApJ, 617, L21

Kaneko, Y., et al. 2006a, ApJS, 166, 298

2006b, in AIP Conf. Proc. 836, Gamma-Ray Bursts in the Swift Era,

ed. S. S. Holt, N. Gehrels, \& J. A. Nousek (Melville: AIP), 133

\section{REFERENCES}

Kumar, P., \& Panaitescu, A. 2000, ApJ, 541, L51

Liang, E., \& Zhang, B. 2005, ApJ, 633, 611

Luckas, P., Trondal, O., \& Schwartz, M. 2005, GCN Circ. 3642, http:// gcn.gsfc.nasa.gov/gcn/gcn3/3642.gcn3

MacLeod, C., et al. 2005, GCN Circ. 3652, http:/gcn.gsfc.nasa.gov/gen/gen3/ 3652.gen3

Mészáros, P., \& Rees, M. 1997, ApJ, 476, 232

Moretti, A., et al. 2006, A\&A, 448, L9

Nakar, E., \& Piran, T. 2005a, MNRAS, 360, L73

2005b, preprint (astro-ph/0503517)

Nemiroff, R. J., et al. 1994, ApJ, 423, 432

Norris, J. P. 2002, ApJ, 579, 386

Norris, J. P., \& Bonnell, J. T. 2006, ApJ, 643, 266

Norris, J. P., Marani, G. F., \& Bonnell, J. T. 2000, ApJ, 534, 248

Norris, J. P., Scargle, J. D., \& Bonnell, J. T. 2001, in Gamma-Ray Bursts in the

Afterglow Era, ed. E. Costa, F. Frontera, \& J. Hjorth (Berlin: Springer), 40

Norris, J. P., et al. 1986, ApJ, 301, 213 1996, ApJ, 459, 393

Nousek, J. A., et al. 2006, ApJ, 642, 389

Palmer, D. M., et al. 2005, Nature, 434, 1107

Panaitescu, A., Mészáros, P., Gehrels, N., Burrows, D., \& Nousek, J. 2006, MNRAS, 366, 1357

Perley, D., et al. 2005, GCN Circ. 3932, http://gcn.gsfc.nasa.gov/gcn/gcn3/ 3932.gcn3

Preece, R. D., et al. 2000, ApJS, 126, 19

Sakamoto, T., et al. 2006, in AIP Conf. Proc. 836, Gamma-Ray Bursts in the Swift Era, ed. S. S. Holt, N. Gehrels, \& J. A. Nousek (Melville: AIP), 43

Sari, R., Piran, T., \& Narayan, R. 1998, ApJ, 497, L17

Yonetoku, D., et al. 2004, ApJ, 609, 935

Zhang, B., \& Mészáros, P. 2004, Int. J. Mod. Phys. A, 19, 2385

Zhang, B., et al. 2006, ApJ, 642, 354 (Z06) 